\title{
Optical encryption and the space bandwidth product
}

\author{
B.M. Hennelly, J.T. Sheridan * \\ Department of Electronic and Electrical Engineering, Faculty of Architecture and Engineering, University College Dublin, \\ Belfield, Dublin 4, Republic of Ireland
}

Received 30 August 2004; received in revised form 19 November 2004; accepted 22 November 2004

\begin{abstract}
The use of optical signal processing in the field of image encryption typically involves the use of optical transforms representing quadratic phase systems (QPS), to implement the optical Fourier transform (OFT), the optical fractional Fourier transform (OFRT), the Fresnel transform (FST) and the general linear Canonical transform (LCT). Random phase keys (RPK) or random shifting stages are applied after transformation and the process is repeated for deeper encryption. Each such stage may change the spatial extent of the complex distribution and may also change the spatial frequency bandwidth of the signal. Therefore, the space bandwidth product (SBP), which is equal to the number of discrete samples that are required to fully represent the encrypted signal (and are required to recover the original), will also change. In general the encrypted field is complex and recording must be carried out using a holographic material or using digital holographic methods. We show how the matrices associated with the effect of a LCT on the Wigner distribution function (WDF) provide us with an efficient method for finding the position, spatial extent, spatial frequency extent and SBP of the encrypted signal. Applying the technique, we review a number of methods proposed in the literature for encryption using optical systems based on the FT, FRT, FST and LCT. This technique also allows us to identify necessary parameters, i.e., we can determine the minimum size of lens apertures and the need for magnification stages at critical stages in the system.
\end{abstract}

(C) 2004 Elsevier B.V. All rights reserved.

PACS: 42.30; 42.30.K; 32.79.S

Keywords: Image processing in optics; Fourier optics; Signal processing in optics

\section{Introduction}

The Fourier transform (FT), fractional Fourier transform (FRT), and Fresnel transform (FST) are all special cases of the linear Canonical transform (LCT). The FT is a linear transformation, which rotates a

\footnotetext{
${ }^{*}$ Corresponding author. Tel.: +3531 716 1927; fax: +35312830921.

E-mail address: john.sheridan@ucd.ie (J.T. Sheridan).
} 
signal in the space domain through $\pi / 2$ radians into the orthogonal spatial frequency domain. Four applications of the FT are equivalent to the identity function. The FRT is a linear transform, which rotates the signal through an arbitrary angle into a mixed frequency-space domain. The fractional order Fourier transform has been used in the field of quantum mechanics [1,2]. In optics it was first applied to describe wave propagation in graded index (GRIN) media [3,4]. It was shown that, while a FT operation could be described as the rotation of the Wigner distribution function (WDF) by an angle of $\pi / 2$, the FRT describes the rotation of the WDF by an angle equal to $a \pi / 2$ where $a$ represents the order of the FRT [5]. Several possible bulk optical implementations were also proposed [4,5]. Since then, the FRT has lead to new applications in many areas where the FT has importance, for example, phase retrieval [6,7], beam shaping [8], filtering [9] and many others, including optical encryption. The FST has the simplest optical implementation of all free space propagation. The FT, FRT, FST and the most general arbitrary LCT have all been applied in the field of optical encryption [10-30].

Information security is of ever increasing importance. Optical signal processing has the distinct advantage of sending 2-D complex data in parallel and carry out otherwise time costly operations at great speeds and they have found growing importance in data encryption. An optical encryption scheme has been proposed [10] dubbed "double random phase encoding" which involves multiplying by two random phase masks (RPK) in the input plane and in the Fourier domain. It can be shown that if these random phases are statistically independent white noises then the encrypted image is also a complex white noise signal. The RPK located at the Fourier plane serves as the only key in this encryption scheme. The properties of this system and systems like it have been extensively investigated [11-13]. The FRT has been utilised in encryption algorithms in conjunction with RPKs [15-21,23] and by randomly shifting sections of the image in some fractional domains $[24,25]$ using the Jigsaw transform (JT). The FST has also been used with RPKs [27-29] and with random shifting applied in some Fresnel domains [29]. The most general form of the LCT, implemented with any arbitrary quadratic phase system has also been used in an encryption system that uses random phase keys [30]. In every one of these cases [10-30] each stage in the encryption process may change the spatial extent and the spatial frequency bandwidth of the complex distribution on the plane normal to the propagation axis. This will result in a change of the signals space bandwidth product (SBP). By analysing the effects of: (i) a LCT, (ii) a Spatial Light Modulator (which can be used to produce a RPK), and (iii) random shifting on the WDF we derive a matrix method for automatically and efficiently determining the position, the spatial width (extent) and spatial frequency bandwidth of the signal at any point in the encryption process including the values of these parameters in the final encrypted image. In general the encrypted image is complex and recording must be carried out using a holographic material or using digital holographic methods. In each case it is desirable to know the spatial extent of the signal to be recorded, its position, and its spatial frequency bandwidth so that we can determine the most efficient way of using the bulk optical system and recording method available. We can deduce the maximum spatial extent and spatial frequency extent of the input image such that the encrypted image can be fully represented after recording. If this is not the case, we can never recover the input image. The method also allows us to determine the minimum size of lens apertures, which will allow us to reduce signal to noise ration (SNR) in the encryption/decryption system and we can also identify the need for magnification at critical stages in the system, i.e., before the signal passes through a SLM or when the signal reaches the camera. In Section 2, we discuss the Wigner distribution function (WDF) and its relationship to the SBP. We will use these concepts in Section 3 to develop the matrix based method.

\section{The space-bandwidth product and the Wigner distribution function}

The WDF of a complex optical amplitude distribution (or Wigner distribution chart) provides a graphical means of simultaneously viewing the signals spatial and spatial frequency distributions and is 
particularly useful for visualising localized signals [31-35]. $W_{u}(x, k)$ the WDF of a signal $u(x)$ defined in terms of its spatial, $x$, distribution is defined in the following way:

$$
W_{u}(x, k)=\psi\{u(x)\}(x, k)=\int_{-\infty}^{\infty} u\left(x-\frac{\xi}{2}\right) u^{*}\left(x-\frac{\xi}{2}\right) \exp (-\mathrm{j} 2 \pi k \xi) \mathrm{d} \xi
$$

where $k$ represents spatial frequency, the asterisk denotes the complex conjugate and $\psi\{u(x)\}(x, k)$ denotes the WDF operator. The real valued WDF doubles the number of dimensions, i.e., a complex one-dimensional signal has a two dimensional WDF while two-dimensional signals have four-dimensional WDFs. In many practical problems it is assumed that a signal is bounded within some finite region in both the spatial and spatial frequency domains. The spatial extent, $W_{0}$ and the frequency extent, $B_{0}$ are defined [35] such that

$$
\begin{aligned}
& u(x) \approx 0 \quad|x|>W_{0} / 2, \\
& U(k)=\int_{-\infty}^{\infty} u(x) \exp (-\mathrm{j} 2 \pi k x) \mathrm{d} x \approx 0 \quad|k|>B_{0} / 2,
\end{aligned}
$$

and therefore, the signal energy is negligible outside these spatial and spatial frequency regions. For all signals discussed here, $W_{0}$ and $B_{0}$ may also be defined as follows [35]:

$$
\int_{-W_{0} / 2}^{W_{0} / 2}|u(x)|^{2} \mathrm{~d} x=\int_{-B_{0} / 2}^{B_{0} / 2}|U(k)|^{2} \mathrm{~d} k=\eta E,
$$

where $\eta$ is less than but approximately equal to 1 and $E$ represents the total signal energy which is

$$
E=\int_{-\infty}^{\infty}|u(x)|^{2} \mathrm{~d} x=\int_{-\infty}^{\infty}|U(k)|^{2} \mathrm{~d} k
$$

Another property of the WDF, which explains the relationship between the WDF of the product of two functions (in space) and the individual WDFs of the two functions, is as follows:

$$
W_{u v}(x, k)=\psi\{u(x) v(x)\}(x, k)=\int_{-\infty}^{\infty} W_{v}(x, k-\xi) W_{u}(x, \xi) \mathrm{d} \xi .
$$

In Fig. 1(i), we show the WDF of a signal $u(x)$ in which the signal energy lies within a rectangular area. The four corner coordinates, which define the shape, are shown on the diagram. The signal $u(x)$ is completely determined if it is sampled equidistantly in $x$ with sample space $\delta x$ such that the Nyquist criteria is satisfied

$$
\delta x \leqslant \frac{1}{B_{0}} .
$$

Therefore, the number of samples, $N$, required to completely describe $u(x)$ is

$$
N=\frac{W_{0}}{\delta x} \geqslant W_{0} B_{0}
$$

Clearly, for the most efficient uniform sampling $\delta x=1 / B_{0}$ and $N=W_{0} B_{0}$ the space-bandwidth product of the signal. In general signals may have an irregular shaped WDF and one such case is shown in Fig. 1(iv). This shape is the result of applying a FST to the signal with the regular WDF shown in Fig. 1. Such a signal can be fully described with a number of samples that is less than the SBP [35] but this requires non-uniform sampling in the space domain.

We note that the use of regular sampling defined using the SBP is of particular significance for optical engineers working in the laboratory, using a CCD camera with a regular periodic pattern of pixels. Such periodic sampling is usually interpreted as corresponding to a regular supporting area in phase space 


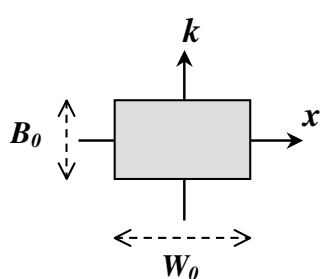

(i)

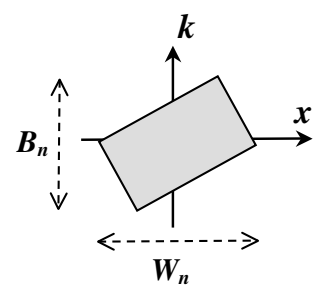

(iii)

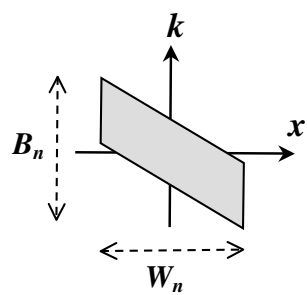

(v)

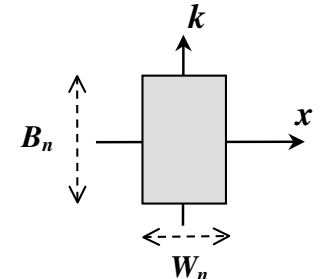

(ii)

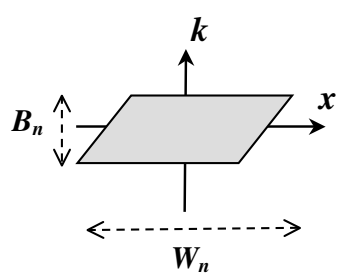

(iv)

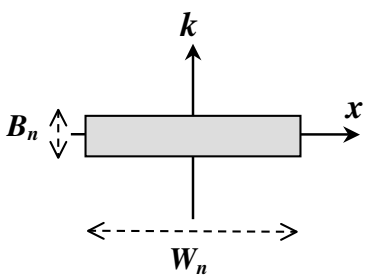

(vi)

Fig. 1. WDF of a signal before and after different types of LCT are applied to the signal: (i) the WDF of the original signal, (ii) the WDF of the signal after it has been Fourier transformed, (iii) the WDF of the signal after it has been fractional Fourier transformed, (iv) the WDF of the Fresnel transformed signal, (v) the WDF of the signal after it has been chirp multiplied, and (vi) WDF of magnified signal.

(rectangular in the 1-D signal case). A skewed rectangle (following for example a Fresnel transformation in the 1-D case) becomes a sub-area inside a larger rectangle, unless parts of the original subtending area are intentionally neglected. Thus, the total number of samples necessary to ensure capture of all the information input to the system will usually involve a change in the regular sampling. Following the standard procedure, we assume sufficient regular sampling to ensure that aliasing effects can be assumed negligible. While some 'over sampling' may occur compared to a situation in which sufficient knowledge is available to allow a prori pre-processing of the data [36], in our method no pre- or post-processing of the data is necessary.

In the following section, we discuss the linear Canonical transform and its effect on the WDF. In particular we analyse its effect on the shape of a bounded area (support) on the WDF. From this discussion we will develop the matrix based technique used to automatically characterize optical encryption systems.

\section{SBP and LCT: the matrix technique}

The 1-D LCT [32,34] is a three-parameter class of linear integral transform and is defined as follows: 


$$
u_{\alpha, \beta, \gamma}\left(x^{\prime}\right)=L_{\alpha, \beta, \gamma}\{u(x)\}\left(x^{\prime}\right)=\exp [-\mathrm{j} \pi / 4] \sqrt{\beta} \int_{-\infty}^{\infty} u(x) \exp \left[\mathrm{j} \pi\left(\alpha x^{2}-2 \beta x x^{\prime}+\gamma x^{\prime 2}\right)\right] \mathrm{d} x,
$$

where $\alpha, \beta$ and $\gamma$ are real transform parameters which are independent of the $x$ and $x^{\prime}$ domain coordinates. This can be further generalised to a five-parameter transform, the special affine Fourier transform [37,38] in which the additional two parameters are shifts in the spatial and spatial frequency domain, which have no effect on the numerics. The LCT is a unitary transform and includes as special cases the FT, the FST, the FRT, and the operations of scaling (magnification) and chirp multiplication (thin lenses). Optical systems implemented using an arbitrary number of thin lenses and propagation through free space in the Fresnel approximation, or through sections of graded-index (GRIN) media, belong to the class of systems known as quadratic-phase systems (QPS) [39]. All QPS can be described mathematically using the LCT. In our formulation, the wavelength factor has been included as a part of $\alpha, \beta$ and $\gamma$. However, in most cases in the literature the wavelength is explicitly given as a parameter in the definition of the QPS, since it is common to all three parameters. The effect of the operator $L_{\alpha, \beta, \gamma}$ on the WDF of the signal is

$$
W(x, k) \rightarrow W(a x+b k, c x+d k),
$$

where $a d-b c=1, a=\gamma / \beta, b=1 / \beta, c=-\beta+\alpha \gamma / \beta$ and $d=\alpha / \beta$. This is equivalent to the following matrix transformation acting in phase space:

$$
\left[\begin{array}{l}
x^{\prime} \\
k^{\prime}
\end{array}\right]=\mathbf{L}\left[\begin{array}{l}
x \\
k
\end{array}\right]=\left[\begin{array}{ll}
a & b \\
c & d
\end{array}\right]\left[\begin{array}{l}
x \\
k
\end{array}\right]=\left[\begin{array}{cc}
\gamma / \beta & 1 / \beta \\
-\beta+\alpha \gamma / \beta & \alpha / \beta
\end{array}\right]\left[\begin{array}{l}
x \\
k
\end{array}\right] .
$$

These matrices are unit determinant implying a conservation of area (energy) on the WDF chart. The matrices are useful because the product of the composition matrices of two or more successive optical systems is the matrix of the overall optical system. We have found further use for these matrices by developing a formalism to automatically calculate the SBP using matrix algebra. In Fig. 2, we show the WDFs of a signal and of its LCT. We have assumed that the signals energy lies within some arbitrary four-sided shape (asymmetrical in $x-k$ ) which is defined by the corner coordinates $\left(x_{1}, k_{1}\right),\left(x_{2}, k_{2}\right),\left(x_{3}, k_{3}\right)$ and $\left(x_{4}, k_{4}\right)$. In fact, our method allows us to take any number of such points and sides to define the bounded area in which most of the signals energy lies. The spatial extent of the signal is $W_{0}$ its spatial frequency bandwidth is $B_{0}$ and the number of samples required to fully describe the signal, with uniform equidistant sampling, is $N_{0}=W_{0} B_{0}$. After applying the LCT the shape changes. However, the bounded area (the bounded energy) and the number of sides remains the same due to the affine nature of the LCT. The number of samples now required to determine the transformed signal, with uniform equidistant sampling, is given by $N_{n}=W_{n} B_{n}$.

Using matrix algebra the change in position of each of the four coordinates defining the WDF, and thus $W_{n}$ and $B_{n}$ and subsequently $N_{n}$ can be simply found as follows. Given

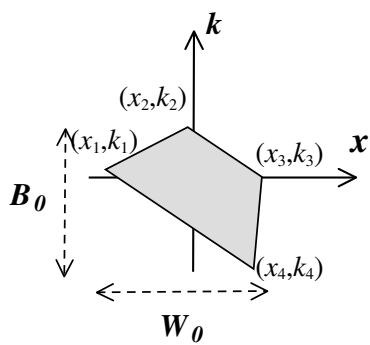

(a)

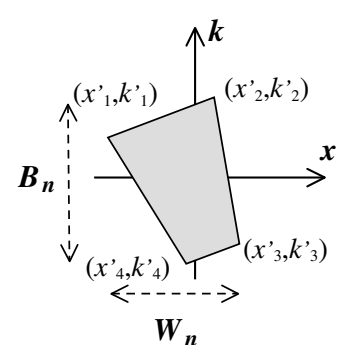

(b)

Fig. 2. WDF of a signal, with bounded enegy, before and after application of an arbitrary LCT. 


$$
\mathbf{S}=\left[\begin{array}{llll}
x_{1} & x_{2} & x_{3} & x_{4} \\
k_{1} & k_{2} & k_{3} & k_{4}
\end{array}\right]
$$

the new corner coordinates are given by

$$
\mathbf{S}^{\prime}=\mathbf{L S}=\left[\begin{array}{llll}
x_{1}^{\prime} & x_{2}^{\prime} & x_{3}^{\prime} & x_{4}^{\prime} \\
k_{1}^{\prime} & k_{2}^{\prime} & k_{3}^{\prime} & k_{4}^{\prime}
\end{array}\right]=\left[\begin{array}{llll}
a x_{1}+b k_{1} & a x_{2}+b k_{2} & a x_{3}+b k_{3} & a x_{4}+b k_{4} \\
c x_{1}+d k_{1} & c x_{2}+d k_{2} & c x_{3}+d k_{3} & c x_{4}+d k_{4}
\end{array}\right],
$$

where $\mathbf{S}$ and $\mathbf{S}^{\prime}$ are the corner coordinate matrices (CCM) before and after application of the LCT. The spatial extent is clearly the maximum distance between any two of the $x$ coordinates, while similarly the spatial frequency bandwidth is the maximum distance between any two of the $k$ coordinates. The spatial extent and the spatial frequency can be automatically obtained from the CCM using the Distances Matrix, D

$$
\mathbf{D}=\left[\begin{array}{cccccc}
1 & 1 & 1 & 0 & 0 & 0 \\
-1 & 0 & 0 & 1 & 1 & 0 \\
0 & -1 & 0 & -1 & 0 & 1 \\
0 & 0 & -1 & 0 & -1 & -1
\end{array}\right]
$$

To illustrate the procedure we find $W_{0}$ and $B_{0}$ of the original signal. We define the Extent Vector, E

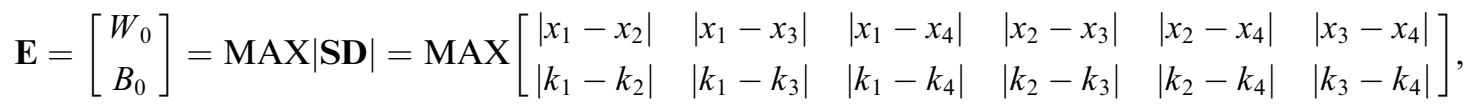

where we have introduced the notation MAX to denote the maximum element in each row. On the righthand side of Eq. (13), we find the distance between each possible pair of coordinates in the $x$ (top row) and the $k$ (bottom row). The absolute value of each element is then calculated giving a $2 \times 6$ matrix of positive elements. The maximum value appearing in each of the two rows is determined giving a $2 \times 1$ vector. The element left in the top row is $W_{0}$ and the element in the bottom row is $B_{0}$. To calculate $N_{0}$ we find the product of the transpose and rotated Extent Vector as shown in Eq. (14) where $\mathbf{R}$ is the Rotation Matrix

$$
N_{0}=\frac{1}{2} \mathbf{E}^{\mathbf{t}} \mathbf{R E}=\frac{1}{2}\left[\begin{array}{ll}
W_{0} & B_{0}
\end{array}\right]\left[\begin{array}{ll}
0 & 1 \\
1 & 0
\end{array}\right]\left[\begin{array}{c}
W_{0} \\
B_{0}
\end{array}\right]=W_{0} B_{0} .
$$

After application of the LCT, the transformed signal has a new Extent Vector, $\mathbf{E}^{\prime}$

$$
\begin{aligned}
& \mathbf{E}^{\prime}=\left[\begin{array}{c}
W_{n} \\
B_{n}
\end{array}\right]=\operatorname{MAX}\left|\mathbf{S}^{\prime} \mathbf{D}\right|=\operatorname{MAX}\left[\begin{array}{ll}
\left|a\left(x_{1}-x_{2}\right)+b\left(k_{1}-k_{2}\right)\right| & \left|a\left(x_{1}-x_{3}\right)+b\left(k_{1}-k_{3}\right)\right| \\
\left|c\left(x_{1}-x_{2}\right)+d\left(k_{1}-k_{2}\right)\right| & \left|c\left(x_{1}-x_{3}\right)+d\left(k_{1}-k_{3}\right)\right|
\end{array}\right. \\
& \left|a\left(x_{1}-x_{4}\right)+b\left(k_{1}-k_{4}\right)\right| \quad\left|a\left(x_{2}-x_{3}\right)+b\left(k_{2}-k_{3}\right)\right| \\
& \left|c\left(x_{1}-x_{4}\right)+d\left(k_{1}-k_{4}\right)\right| \quad\left|c\left(x_{2}-x_{3}\right)+d\left(k_{2}-k_{3}\right)\right| \\
& \left.\begin{array}{ll}
\left|a\left(x_{2}-x_{4}\right)+b\left(k_{2}-k_{4}\right)\right| & \left|a\left(x_{3}-x_{4}\right)+b\left(k_{3}-k_{4}\right)\right| \\
\left|c\left(x_{2}-x_{4}\right)+d\left(k_{2}-k_{4}\right)\right| & \left|c\left(x_{3}-x_{4}\right)+d\left(k_{3}-k_{4}\right)\right|
\end{array}\right] .
\end{aligned}
$$

The largest element in the two rows in the matrix are clearly dependent on the values of $a, b, c$ and $d$ (and therefore the parameters, $\alpha, \beta$ and $\gamma$, of the LCT in question). Once these maximum values are determined it is then possible to calculate the number of equidistant samples required to fully describe the signals. We note that extension to two dimensions, as is necessary for image encryption, can be carried out by treating each of the two dimensions separately and requires no further discussion.

There are many optical encryption schemes which use special cases of the LCT, namely the FT, the FRT, and the FST. Defining the FT in terms of the LCT parameters gives $\alpha=\gamma=0, \beta=1 / \lambda f$ where $f$ is the focal 
length of the Fourier transforming lens and $\lambda$ represents wavelength. Application of the FT causes a rotation of the WDF by $\pi / 2$ radians. This is illustrated in Fig. 1(ii) where we show the WDF of the Fourier transform of the original signal whose (rectangular symmetric) WDF, with spatial extent $W_{0}$ and spatial frequency bandwidth $B_{0}$ is shown in Fig. 1(i). To define the optical FRT we set $\alpha=\gamma=1 /[q \tan (p \pi / 2)]$ and $\beta=1 /[\lambda q \sin (p \pi / 2)]$, where $q$ is called the standard focal length [4] and is dependent on the physical parameters of the optical FRT system, and $p$ is the order of the FRT and defines the domain, $x_{\mathrm{p}}$ into which it transforms. The FRT is defined separately for $p$ equal to integer multiples of 2 . When $p=1$ the FRT reduces to the FT. Application of the FRT of a given order causes a rotation of the WDF by $p \pi / 2$ radians [4]. Such a rotation is illustrated in Fig. 1(iii). The FRT has many optical implementations involving the use of one or more lenses and sections of free space. Defining the FST in terms of the LCT gives $\alpha=\gamma=\beta=1 / \lambda z$ where $z$ is the distance propagated. Application of the FST of a given distance causes a horizontal shearing of the WDF in the space dimension (the amount of shearing is dependent on the value of $z$ ). This effect is illustrated in Fig. 1(iv). Chirp multiplication which describes the action of a thin lens is defined by $a=d=1$, $b=0$, and $\mathrm{c}=1 / \lambda f$. The effect of chirp multiplication is shown in Fig. 1(v). Scaling, with a magnification factor $M$, is defined by setting $a=M, d=1 / M$, and $b=c=0$ and the effect on the WDF is shown in Fig. 1(vi). We note that, except for the FT and scaling, all of these transforms cause a change in the SBP (by changing the spatial extent and/or the spatial frequency extent).

Earlier we noted that we can take any number of coordinates and sides to define the initial bounded area in which most of the signals energy lies. In relation to this, when the WDF is bounded by $n$ sides (using $n$ coordinates) the Corner Coordinate Matrix, $\mathbf{S}$, will be of dimension $2 \times n$, and the Distances Matrix, $\mathbf{D}$, will be of dimension $n \times\left(\sum_{i=1}^{n-1} i\right)$. Extension to more than four corner coordinates is therefore straightforward.

In this section, we have derived a matrix method to efficiently determine the effect of any optical transformation on the shape of a signals WDF. This allows us to find the position of the signals extent in space, the size of this spatial extent, the position of the spatial frequency bandwidth and the size of this bandwitdth. This gives us the SBP of the signal after optical transformation. In the following section, we discuss the RPK and its effect on the WDF shape (in terms of its effect on the Corner Coordinate Matrix) and therefore on the SBP.

\section{The WDF representation and random phase keys}

In this paper, we assume that every RPK used in the various encryption schemes are implemented by using a spatial light modulator (SLM) which is made up of a finite number, $N$, of pixels of variable refractive index. We assume also that the pixels are set to represent a stationary white noise signal. In Fig. 3, we

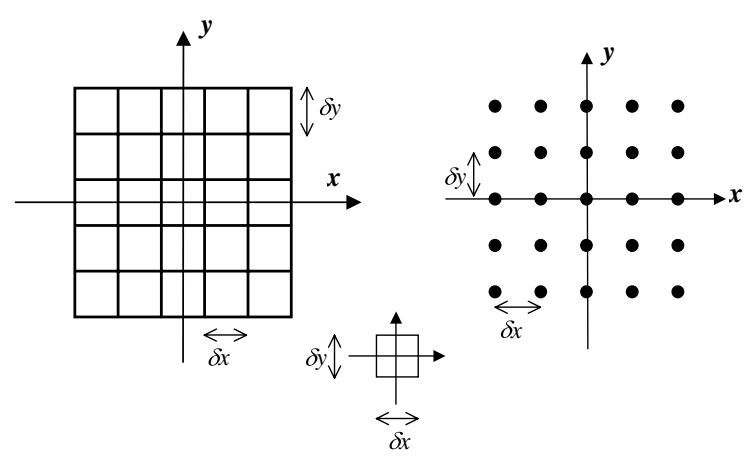

Fig. 3. A spatial light modulator as a convolution of one pixel area with the sample values. 


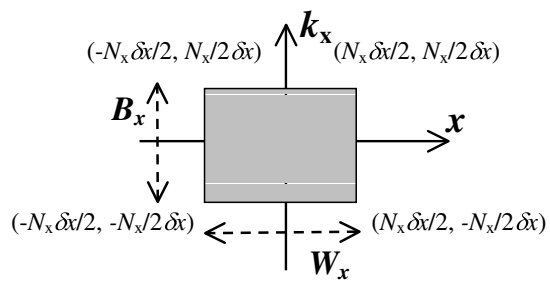

(a)

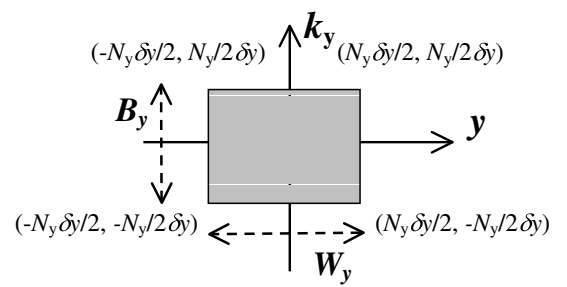

(b)

Fig. 4. The WDF of the centered random phase mask shown separately: (a) for $x$ direction and (b) for $y$ direction.

show how a SLM can be described by the convolution of a rect function, with the dimensions of one pixel, with a sampled random function, where each sample is given by a Dirac delta function, which has been modulated by some random phase value.

Convolution in the spatial domain is equivalent to multiplication in the spatial frequency domain. Since we can assume that the size of $\operatorname{Rect}(\delta x, \delta y)$ will be small, we can assume that its FT, a sinc function, will have a very large extension. The spatial frequency extension of the randomized SLM is will be given by $N_{x} /$ $\delta x$ in the $x$ direction and $N_{y} / \delta y$ where $N_{x}$ and $N_{y}$ are the number of pixels in the $x$ and $y$ directions, respectively. We will assume that the SLM is perfectly centered on the axis of propagation. The WDF of this randomized SLM is four dimensional, however, for this discussion it suffices to deal with the WDF separately for $x$ and $y$ as shown in Fig. 4.

When a wave field passed through the SLM the wavefield and the SLM random function are multiplied in the spatial domain. Eq. (5) shows how we can relate the WDFs of the two signals. To find the WDF of the new signal we convolve the two component WDFs along the frequency axes, i.e., separately in $k_{x}$ and $k_{y}$. We must always assume that the spatial extent of the random phase (SLM) is greater than or equal to that of the wavefield passing through it. The SLM is used to ensure that the CCM of the signal lies within the region of the WDF occupied by the SLM. This may require magnification we will show in Section 6 how our method can account for this. This convolution is shown in Fig. 5 for the $x$ direction. On the left of Fig. 5, we show the two WDFs as they convolve along the $k$ axis, the square one is the random phase, and the centred one corresponds to a Fresnel transformed signal. On the right we show the shape on the WDF within which the signals energy is localised.

Through successive applications of a random phase screen there will be further increases in the number of coordinates in the CCM. Taking the input CCM to be defined by Eq. (11.1), we define the following operation on the CCM vector to describing the approximate effect of a SLM. First we find the Extent Vector of the signal using Eq. (13) and this gives us values for $W_{x}$ and $B_{x}$ then we carry out the following change in $\mathbf{S}$ :
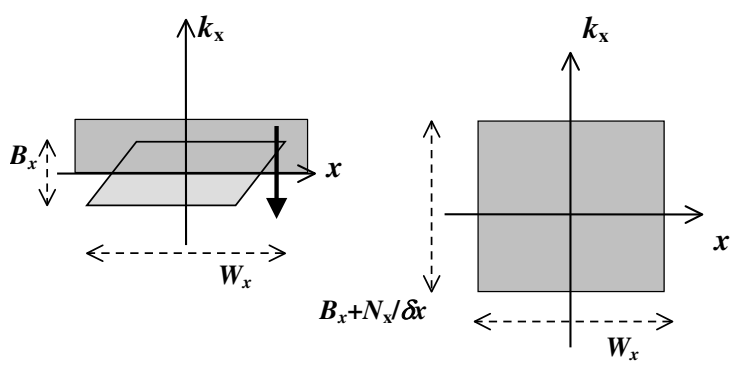

Fig. 5. Convolution (in $k$ ) of the WDFs of the RPK and the input (Fresnel transformed) wave field. 


$$
\left[\begin{array}{llll}
x_{1} & x_{2} & x_{3} & x_{4} \\
k_{1} & k_{2} & k_{3} & k_{4}
\end{array}\right] \rightarrow\left[\begin{array}{cccc}
-W_{x} / 2 & W_{x} / 2 & W_{x} / 2 & -W_{x} / 2 \\
B_{x} / 2+N / 2 \delta x & B_{x} / 2+N / 2 \delta x & -B_{x} / 2-N / 2 \delta x & -B_{x} / 2-N / 2 \delta x
\end{array}\right] .
$$

The true shape of the WDF will be somewhat more complicated and the number of coordinates defining the new CCM will double each time the signal passes through a SLM. This WDF is difficult to define analytically in the general case but can be easily defined for any specific case by convolving the WDF shapes along the frequency axis.

An identical argument can be applied separately to the $y$ direction. We see from Fig. 5 that the spatial extent of the signal will not change but the spatial frequency bandwidth will increase by an amount equal to that of the bandwidth of the random SLM. In the later analysis the change in the CCM, S, brought about by the SLM with parameters $\delta x$ and $N$ and defined by Eq. (16) will be denoted as follows $\operatorname{SLM}_{\delta x, N}\{\mathbf{S}\}$. The above analysis is also true for the $y$ direction.

An alternative to using the RPK in encryption process is the JT. Thus, far we discussed the effect of optical transformations and RPKs on shapes in the WDF and also on the SBP. In the following section, we discuss the effect of the JT in the same context.

\section{The Jigsaw transform and the WDF}

In a number of encryption systems the JT $[15,26,29], J\{\}$, which juxtaposes different sections of the complex image is used after application of some optical transformation. We show the effect of this transform on our input image in Fig. 6. In this case the image is broken up into 64 subsections of $8 \times 8$ pixels, which were repositioned relative to each other according to some permutation. This can be extended to juxtaposing individual pixels. The permutation used is random. The JT is unitary, energy being conserved through the transform and it also has an inverse. In the case shown in Fig. 6, there are 64! possible JT permutations. Each JT is denoted by an index, e.g., $J_{\mathrm{b}}\{\}$ and its inverse is denoted by $J_{-\mathrm{b}}\{\}$.

Shifting occurs along both dimensions and in Fig. 7, we show the WDF of a single row of pixels (after Fresnel transformation) along the $x$ direction for any arbitrary value of $y$. Due to the non-linearity of the WDF it is impossible to predict the new shape of the WDF. We can, however, determine with certainty that the energy will be contained within a square shape with the same spatial width and frequency bandwidth as before. This is a consequence of the fact that we represent the Jigsaw Transformed signal with some number of uniformly distributed sample values.

We are now in a position to discuss the effect of the JT on the CCM of the signal, i.e., $J_{\mathrm{b}}\{\mathbf{S}\}$. Taking the input CCM to be defined by Eq. (11.1), we define the following operation on the CCM vector to describe the effect of the Jigsaw on the CCM. First we find the Extent Vector of the signal using Eq. (13) and this gives us values for $W_{0}$ and $B_{0}$. Then we carry out the following change in $\mathbf{S}$ :

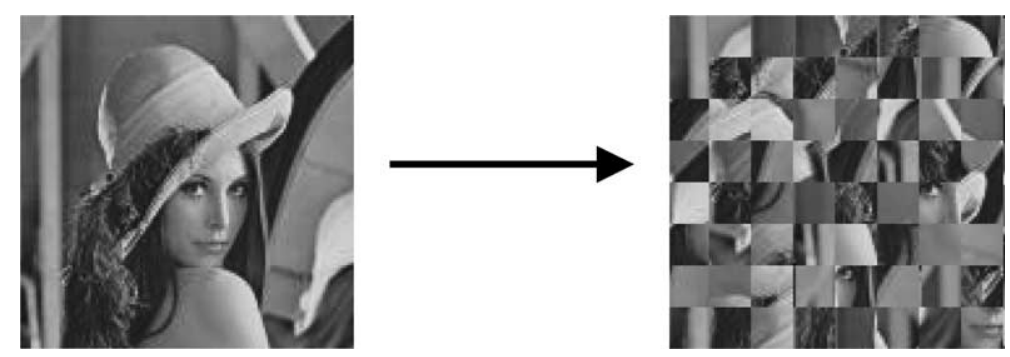

Fig. 6. The Jigsaw transform applied to a Lena image. 


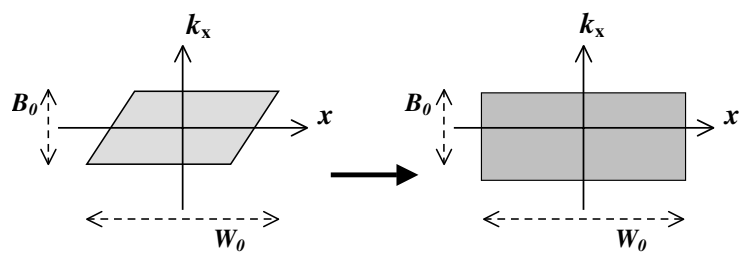

Fig. 7. The effect of the Jigsaw transform on the WDF of a row of pixels in the $x$ direction.

$$
\left[\begin{array}{llll}
x_{1} & x_{2} & x_{3} & x_{4} \\
k_{1} & k_{2} & k_{3} & k_{4}
\end{array}\right] \rightarrow\left[\begin{array}{cccc}
-W_{0} / 2 & W_{0} / 2 & W_{0} / 2 & -W_{0} / 2 \\
B_{0} / 2 & B_{0} / 2 & -B_{0} / 2 & -B_{0} / 2
\end{array}\right] .
$$

The same analysis can be applied in the $y$ direction.

\section{The SBP and encryption algorithms}

\subsection{Method 1 [10]}

We begin this section reviewing the first Fourier based optical encryption scheme presented in [10] which uses the FT. Two RPKs are used in the encryption scheme, which are in the form of two statistically independent white sequences uniformly distributed in $[0,1]$. We will denote these random functions as $n_{1}$ and $n_{2}$. Initially, the input image to be encoded is multiplied by one RPK. Then applying a FT using a convex lens and in the Fourier domain, it is multiplied by the second RPK. Finally we apply a second FT. This is equivalent to a convolution operation, where the encrypted image can be represented by

$$
g(x)=\left\{f(x) \exp \left[\mathrm{j} 2 \pi n_{1}(x)\right]\right\} * h(x),
$$

where the $*$ denotes the convolution operation and $f(x)$ represents the signal to be encrypted. We note the use of 1-D functions for simplicity

$$
F\{h(x)\}=\exp \left[\mathrm{j} 2 \pi n_{2}\left(k_{x}\right)\right] .
$$

The resulting encrypted image is a stationary white noise. The first RPK serves to make the input image white but non-stationary and not encrypted. The second serves to make the image stationary and encoded. Because the encrypted image is complex valued, both the real and imaginary parts are needed to decode the image. In order to record such a signal, we must use holographic methods. To decrypt we apply the inverse of what was done to encrypt the image: (i) first we return to the Fourier domain through the action of a lens; (ii) then comes multiplication by a RPK, which is the conjugate of the corresponding RPK used in the encryption process; (iii) one last Fourier transforming lens follows this. The resulting wave field will have an amplitude distribution equal to the original image so holographic techniques are not necessary to capture it. The only key in this encryption scheme is the second RPK. An optical encryption/decryption implementation can be seen in Fig. 2. The SLM can display both amplitude and phase information. For encryption, SLM1 displays the input image multiplied by the first random phase, while SLM2 displays the second RPK. For decryption, SLM1 displays the encrypted image and SLM2 displays the conjugate of the second RPK.

Note, that we do not need to record the complex decrypted image since we only require its intensity. The properties of such an encryption system have been investigated in detail [17,18,21]. This optical encoding scheme has also been extended to use a phase (only) modulated signal as the input to the system instead of amplitude based image [19], this produces an improvement in robustness to additive noise but the first 
phase mask must be included in decryption and the final decrypted image must be recorded using holographic methods.

If the input function is defined by a spatial width $W_{0}$ and a spatial frequency bandwidth $B_{0}$ (with $N_{0}=W_{0} B_{0}$ if the image is represented digitally), then the input CCM is given by

$$
S=\left[\begin{array}{cccc}
-W_{0} / 2 & W_{0} / 2 & W_{0} / 2 & -W_{0} / 2 \\
B_{0} / 2 & B_{0} / 2 & -B_{0} / 2 & -B_{0} / 2
\end{array}\right]
$$

To find the CCM of the encrypted image we write

$$
\mathbf{S}^{\prime}=\left[\begin{array}{cc}
0 & \lambda f \\
-1 / \lambda f & 0
\end{array}\right] \operatorname{SLM}_{\delta x, N}\left\{\left[\begin{array}{cc}
0 & \lambda f \\
-1 / \lambda f & 0
\end{array}\right] \operatorname{SLM}_{\delta x, N}\{\mathbf{S}\}\right\} .
$$

The Extent Vector of the encrypted image is calculated by Eq. (13) and we now know the position, the spatial width, the spatial frequency bandwidth and the SBP of the encrypted signal which will enable us to choose equipment and resolution at the input to optimise the system.

Furthermore, we can improve upon our system by calculating minimum lens apertures and placing an iris at these points to remove stray noise and we can add magnification stages so that the incident wavefield has had the same spatial extent as the SLM screen. To do this, we rewrite Eq. (21), replacing the OFT matrices with the matrices for its bulk optical implementation, i.e., Fresnel propagation a distance $f$, followed by a lens of focal length $f$, followed by a second Fresnel propagation of distance $f$

$$
\begin{aligned}
\mathbf{S}^{\prime} & =\left[\begin{array}{cc}
1 & \lambda f \\
0 & 1
\end{array}\right]\left[\begin{array}{cc}
1 & 0 \\
-1 / \lambda f & 1
\end{array}\right]\left[\begin{array}{cc}
1 & \lambda f \\
0 & 1
\end{array}\right] \operatorname{SLM}_{\delta x, N}\left\{\left[\begin{array}{cc}
1 & \lambda f \\
0 & 1
\end{array}\right]\left[\begin{array}{cc}
1 & 0 \\
-1 / \lambda f & 1
\end{array}\right]\left[\begin{array}{cc}
1 & \lambda f \\
0 & 1
\end{array}\right] \operatorname{SLM}_{\delta x, N}\{\mathbf{S}\}\right\} \\
& =\mathbf{M}_{3} \mathbf{M}_{2} \mathbf{M}_{1} \mathbf{S L M}_{\delta x, N}\left\{\mathbf{M}_{3} \mathbf{M}_{2} \mathbf{M}_{1} \operatorname{SLM}_{\delta x, N}\{\mathbf{S}\}\right\} .
\end{aligned}
$$

Taking $\mathbf{S}_{1}=\mathbf{M}_{3} \mathbf{M}_{2} \mathbf{M}_{1} \operatorname{SLM}_{\delta x, N}\{\mathbf{S}\}$ to be the CCM of the signal just after application of the first OFT we can now determine the Extent Vector of the CCM, which will provide us with a value of this signals spatial extent, $W_{1}$ and spatial frequency bandwidth $B_{1}$. Comparing $W_{1}$ with the size of the SLM screen, $N \delta x$ we can introduce a magnification stage to match the spatial widths.

We now rewrite our expression for $\mathbf{S}^{\prime}$

$$
\begin{aligned}
\mathbf{S}^{\prime}= & {\left[\begin{array}{ll}
1 & \lambda f \\
0 & 1
\end{array}\right]\left[\begin{array}{cc}
1 & 0 \\
-1 / \lambda f & 1
\end{array}\right]\left[\begin{array}{cc}
1 & \lambda f \\
0 & 1
\end{array}\right] } \\
& \times \operatorname{SLM}_{\delta x, N}\left\{\left[\begin{array}{cc}
M_{1} & 0 \\
0 & 1 / M_{1}
\end{array}\right]\left[\begin{array}{cc}
1 & \lambda f \\
0 & 1
\end{array}\right]\left[\begin{array}{cc}
1 & 0 \\
-1 / \lambda f & 1
\end{array}\right]\left[\begin{array}{cc}
1 & \lambda f \\
0 & 1
\end{array}\right] \operatorname{SLM}_{\delta x, N}\{\mathbf{S}\}\right\} \\
= & \mathbf{M}_{3} \mathbf{M}_{2} \mathbf{M}_{1} \operatorname{SLM}_{\delta x, N}\left\{\mathbf{M}_{4} \mathbf{M}_{3} \mathbf{M}_{2} \mathbf{M}_{1} \operatorname{SLM}_{\delta x, N}\{\mathbf{S}\}\right\},
\end{aligned}
$$

where the magnification factor $M_{1}=N \delta x / W_{1}$. Now we can compute the Extent Vector of $\mathbf{S}^{\prime}$ which will have a spatial extent value $W_{2}$ and a spatial frequency bandwidth $B_{2}$. Taking the camera width or region of holographic material available to be $W_{\text {cam }}$ we include a second magnification stage with to match these spatial widths.

$$
\begin{aligned}
\mathbf{S}^{\prime}= & {\left[\begin{array}{cc}
M_{2} & 0 \\
0 & 1 / M_{2}
\end{array}\right]\left[\begin{array}{cc}
1 & \lambda f \\
0 & 1
\end{array}\right]\left[\begin{array}{cc}
1 & 0 \\
-1 / \lambda f & 1
\end{array}\right]\left[\begin{array}{cc}
1 & \lambda f \\
0 & 1
\end{array}\right] } \\
& \times \operatorname{SLM}_{\delta x, N}\left\{\left[\begin{array}{cc}
M_{1} & 0 \\
0 & 1 / M_{1}
\end{array}\right]\left[\begin{array}{cc}
1 & \lambda f \\
0 & 1
\end{array}\right]\left[\begin{array}{cc}
1 & 0 \\
-1 / \lambda f & 1
\end{array}\right]\left[\begin{array}{cc}
1 & \lambda f \\
0 & 1
\end{array}\right] \operatorname{SLM}_{\delta x, N}\{\mathbf{S}\}\right\} \\
= & \mathbf{M}_{5} \mathbf{M}_{3} \mathbf{M}_{2} \mathbf{M}_{1} \operatorname{SLM}_{\delta x, N}\left\{\mathbf{M}_{4} \mathbf{M}_{3} \mathbf{M}_{2} \mathbf{M}_{1} \operatorname{SLM}_{\delta x, N}\{\mathbf{S}\}\right\},
\end{aligned}
$$


where the magnification factor $M_{2} W_{\text {cam }} / W_{2}$. Now we can compute the Extent Vector of $\mathbf{S}^{\prime}$ which will have a spatial extent value $W_{2}$ and a spatial frequency bandwidth $B_{2}$. To find the minimum size of the first lens aperture, we find the CCM of the signal just before it passes through the lens $\mathbf{S}_{3}=\mathbf{M}_{1} \mathrm{SLM}_{\delta x, N}\{\mathbf{S}\}$. We compute the Extent Vector which gives us a value for the spatial extent of this signal which we denote $W_{x}$. We carry out the same procedure for the $y$ direction to find $W_{y}$. The minimum lens aperture is given by $A=\sqrt{W_{x}^{2}+W_{y}^{2}}$. We repeat this procedure to find the minimum aperture of the second lens.

From Eq. (23), we have an expression relating the CCMs of the input to and the output from the encryption system, which gives us an expression for the maximum possible input signal SBP such that our recording equipment can fully record the encrypted signal and allow for complete recovery of the input signal after decryption. This allows us to determine the maximum spatial frequency bandwidth of the input signal that will be recoverable.

\subsection{Method 2 [15]}

In [15], the authors propose an optical encryption scheme very similar to the one described above, making use of the extra degree of freedom offered by the FRT. Fig. 8 represents the encryption and decryption schemes. The lenses in this diagram now represent optical FRT operations. For the encryption process, the first lens represents a FRT operation of order $a_{1}$ and the second lens represents a FRT operation of order $a_{2}$. For the decryption process, the first lens represents a FRT operation of order $-a_{2}$ and the second lens represents a FRT operation of order $a_{1}$. Again, two phase masks are used which are in the form of two statistically independent white sequences uniformly distributed in $[0,1]$. The encryption scheme is as follows; the input image to be encoded is multiplied by one RPK to give us $f(x) \exp \left[\mathrm{j} \pi n_{1}(x)\right]$. A FRT operation of order $p_{1}$ was applied through a convex lens to give, $F_{\mathrm{p} 1}\left\{f(x) \exp \left[\mathrm{j} \pi n_{1}(x)\right]\right\}$. Now, in this fractional domain, the image is multiplied by the second RPK to give $F_{\mathrm{p} 1}\left\{f(x) \exp \left[\mathrm{j} \pi n_{1}(x)\right]\right\} \exp \left[\mathrm{j} \pi n_{2}(x)\right]$. The resulting image is again transformed by a FRT operation, this time of order $p_{2}$, through the use of a second lens to provide us with our encrypted image $g(x)$

$$
g(x)=F_{\mathrm{p} 2}\left\{F_{\mathrm{p} 1}\left\{f(x) \exp \left[\mathrm{j} 2 \pi n_{1}(x)\right]\right\} \exp \left[\mathrm{j} 2 \pi n_{2}(x)\right]\right\} .
$$

The result is that we have buried or hidden our phase key in some fractional domain. It is shown [20] that the result of this method of encryption is to encode our input signal into a white stationary noise. Once again, decryption is the exact inverse of encryption. An optical method was proposed to implement this algorithm [21]. Taking the input CCM to the system as being defined by Eq. (20) the CCM of the encrypted image is

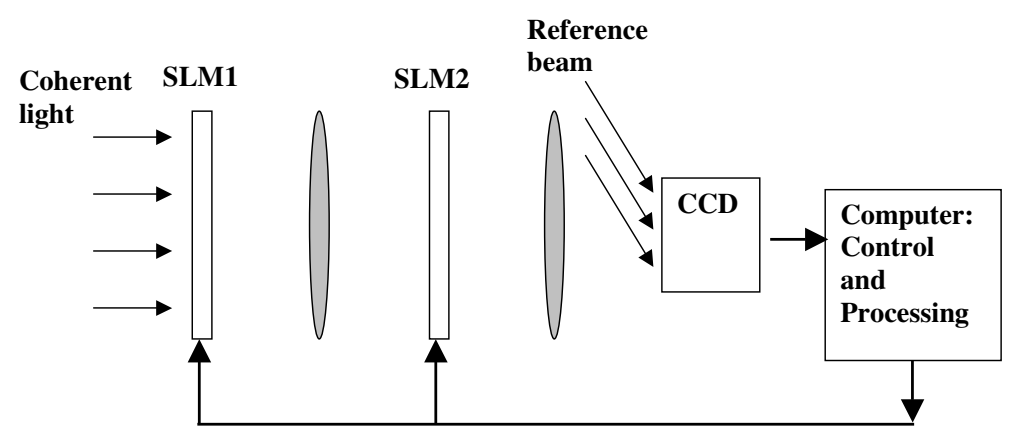

Fig. 8. General optical encryption/decryption set-up for method 1 and method 2. 


$$
\mathbf{S}^{\prime}=\left[\begin{array}{cc}
\cos \left(p_{2} \pi / 2\right) & \lambda q \sin \left(p_{2} \pi / 2\right) \\
-\sin \left(p_{2} \pi / 2\right) \lambda q & \cos \left(p_{2} \pi / 2\right)
\end{array}\right] \operatorname{SLM}_{\delta x, N}\left\{\left[\begin{array}{cc}
\cos \left(p_{1} \pi / 2\right) & \lambda q \sin \left(p_{1} \pi / 2\right) \\
-\sin \left(p_{1} \pi / 2\right) / \lambda q & \cos \left(p_{1} \pi / 2\right)
\end{array}\right] \operatorname{SLM}_{\delta x, N}\{\mathbf{S}\}\right\} .
$$

The Extent Vector of the encrypted image is calculated using Eq. (13) and we now know the position, the spatial width, the spatial frequency bandwidth and the SBP of the encrypted signal which will enable us to determine suitable choices of equipment and resolution to optimise the system. To determine necessary magnification stages and minimum lens aperture sizes we would decompose the FRT matrices into the component matrices of the individual optical elements (which depends on which FRT optical implementation we use) and carry out a similar procedure to that presented in Section 6.1.

\subsection{Method 3 [30]}

In [30] technique based on a random shifting or JT, applied in FRT domains is proposed. The main advantage of this algorithm is that we do not need to use any phase keys in order to decrypt the image and yet we encrypt the image in a very similar way. First, the input image is multiplied by a random phase function giving us $f(x) \exp [j \pi n(x)]$ where $n(x)$ is a white sequences uniformly distributed in $[0,1]$. Each particular JT is denoted by some index, e.g., $J_{\mathrm{b}}\{\}$ and its inverse is denoted by $J_{-\mathrm{b}}\{\}$. The JT is applied to our random phased input image to give $J_{-\mathrm{b}}\{f(x) \exp [\mathrm{j} \pi n(x)]\}$. The resulting complex information can be displayed using SLMs, which are able to both the phase and intensity of a waveform. Now we apply a FRT operation of order $a_{1}$ which gives us $F_{\mathrm{a} 1}\left\{J_{-\mathrm{b}}\{f(x) \exp [\mathrm{j} \pi n(x)]\}\right\}$. This complex data is collected using interofemetric methods and a second JT with permutation $b_{2}$ is now applied. The result is $J_{-2}\left\{F_{\mathrm{a} 1}\left\{J_{-\mathrm{b}}\{f(x) \exp [\mathrm{j} \pi n(x)]\}\right\}\right\}$. Again this complex data can be represented using SLMs. A second FRT, this time of order $a_{2}$ is now applied to give $F_{\mathrm{a} 2}\left\{J_{-2}\left\{F_{\mathrm{a} 1}\left\{J_{-\mathrm{b}}\{f(x) \exp [\mathrm{j} 2 \pi n(x)]\}\right\}\right\}\right\}$. Once again, the data can be collected using holographic methods. Applying a third JT, displayed the result on the SLM and a third and final FRT is applied, this time of order $a_{3}$ to give us the encrypted image

$$
g(x)=F_{\mathrm{a} 3}\left\{J_{\mathrm{b} 3}\left\{F_{\mathrm{a} 2}\left\{J_{\mathrm{b} 2}\left\{F_{\mathrm{a} 1}\left\{J_{\mathrm{b} 1}\{f(x) \exp [\mathrm{j} 2 \pi n(x)]\}\right\}\right\}\right\}\right\}\right\} .
$$

We could of course continue applying FRT and JT to further encrypt our image but practical difficulties, in terms of time taken and susceptibility to noise and error, would increase. Decryption is given by the operation

$$
f(x)=J_{-b 1}\left\{F_{-\mathrm{a} 1}\left\{J_{-\mathrm{b} 2}\left\{F_{-\mathrm{a} 2}\left\{J_{-\mathrm{b} 3}\left\{F_{-\mathrm{a} 3}\{g(x)\}\right\}\right\}\right\}\right\}\right\},
$$

and is simply the inverse of the encryption process. At the final stage we need only capture the intensity information since this is our original image. Since the phase of the decrypted signal should be equal to the random phase we originally added to our input image, it can be discarded since it no longer serves any purpose. Without this initial phase, the JT scheme would not an advisable encryption method because it might be possible to recognize high frequency discontinuities and thus break the Jigsaw encryption process. However, the inclusion of the random phase at the beginning serves to whiten the image. Therefore, no obvious sharp discontinuities will occur in the image because because of the juxtaposition of the image pieces. The decryption process described requires the knowledge of nine keys in total. These nine keys are made up of six FRT order keys (three in $x$ and three in $y$ ) and three JT permutations. The JT are applied digitally. SLMs are used to display the signal after each step in the encryption/decryption process a single lens configuration is used to implement the FRT. A reference beam is employed to record the complex data after each FRT operation. We note that in the final stage of the decryption process we do not need the reference beam. Taking the input CCM to the system, defined by Eq. (20), we find the CCM at each of the three stages of the encryption process as follows: 


$$
\begin{aligned}
& \mathbf{S}_{1}=\left[\begin{array}{cc}
\cos \left(a_{1} \pi / 2\right) & \lambda q \sin \left(a_{1} \pi / 2\right) \\
-\sin \left(a_{1} \pi / 2\right) / \lambda q & \cos \left(a_{1} \pi / 2\right)
\end{array}\right] \mathrm{JIG}\left\{\operatorname{SLM}_{\delta x, N}\{\mathbf{S}\}\right\}, \\
& \mathbf{S}_{2}=\left[\begin{array}{cc}
\cos \left(a_{2} \pi / 2\right) & \lambda q \sin \left(a_{2} \pi / 2\right) \\
-\sin \left(a_{2} \pi / 2\right) / \lambda q & \cos \left(a_{2} \pi / 2\right)
\end{array}\right] \mathrm{JIG}\left\{\mathbf{S}_{1}\right\}, \\
& \mathbf{S}_{3}=\left[\begin{array}{cc}
\cos \left(a_{3} \pi / 2\right) & \lambda q \sin \left(a_{3} \pi / 2\right) \\
-\sin \left(a_{3} \pi / 2\right) / \lambda q & \cos \left(a_{3} \pi / 2\right)
\end{array}\right] \mathrm{JIG}\left\{\mathbf{S}_{2}\right\} .
\end{aligned}
$$

The Extent Vector of the of the signal at each stage is calculated by applying Eq. (13) to $\mathbf{S}_{1}, \mathbf{S}_{2}$, and $\mathbf{S}_{3}$ and we now know the position, the spatial width, the spatial frequency bandwidth and the SBP of signal at each stage, enabling us to optimise the system.

A similar method can to applied to all of the optical algorithms, which uses the LCT and its special cases - the FT [10-14], the FRT [15-26], and the FST [27-29]. To determine necessary magnification stages and minimum lens aperture sizes we would decompose the FRT matrices into the component matrices of the individual optical elements (which depends on which FRT optical implementation) and carry out the same procedure as before.

\section{Conclusions}

In this paper, we have examined optical encryption systems, which use random phase keys or masks (RPK) implemented using spatial light modulators (SLM), or random shifting stages, i.e., the Jigsaw transform (JT), in addition to optical transforms based on quadratic phase systems (QPS). The QPS examined include the optical Fourier transform (OFT), the optical fractional Fourier transform (OFRT), the Fresnel transform (FST) and the most general linear Canonical transform (LCT).

We have shown, using the Wigner distribution function (WDF), that at each stage of the encryption process the spatial extent, $W$, of the complex distribution on the plane normal to the propagation axis can change as may change the frequency bandwidth, $B$, of the signal. Therefore, the space bandwidth product (SBP), which is equal to the number of discrete samples, $N=B W$, that are required to fully represent our signal, may also change.

In general the encrypted image is complex and recording must be carried out using digital holography. We have presented an automatic method, based on matrices that act in phase space on the WDF, to determine the spatial extension of the signal to be recorded, its position, and its spatial frequency extents or widths. In this way we can determine which camera will be sufficient to capture the signal based on its SBP value or we can apply reverse engineering to find the maximum input image resolution to suit whatever camera we have available. We can also clearly examine the effects of apertures in the optical system in a clear and consistent manner and define any necessary magnification stages in the system to shape the size of the spatial extent of the wavefield to match SLM or camera extents.

\section{Acknowledgements}

We acknowledge the support of Enterprise Ireland and Science Foundation Ireland through the Research Innovation Fund and the Basic Research Programme, and of the Irish Research Council for Science, Engineering and Technology. 


\section{References}

[1] V. Namias, J. Inst. Math. Appl. 25 (1980) 241.

[2] A.C. McBride, F.H. Kerr, IMA. J. Appl. Math. 39 (1987) 159.

[3] D. Mendlovic, H.M. Ozaktas, J. Opt. Soc. Am. A 10 (1993) 1875.

[4] H.M. Ozaktas, D. Mendlovic, J. Opt. Soc. Am. A 10 (1993) 2522.

[5] A.W. Lohmann, J. Opt. Soc. Am. A 10 (1993) 2181.

[6] Z. Zalevsky, D. Mendlovic, R.G. Dorsch, Opt. Lett. 21 (12) (1996) 842.

[7] W.-X. Cong, N.-X. Chen, B.-Y. Gu, Appl. Opt. 37 (29) (1998) 6906.

[8] Y. Zhang, B. Dong, B. Gu, G. Yang, J. Opt. Soc. Am. A 15 (5) (1998) 1114.

[9] H.M. Ozaktas, B. Barshan, D. Mendlovic, L. Onural, J. Opt. Soc. Am. A 11 (1994) 547.

[10] P. Refregier, B. Javidi, Opt. Lett. 20 (7) (1995) 767.

[11] B. Wang, C.C. Sun, W.C. Su, A.E.T. Chiou, Appl. Opt. 39 (26) (2000) 4788.

[12] F. Goudail, F. Bollaro, B. Javidi, P. Refregeir, J. Opt. Soc. Am. A 15 (10) (1998) 2629.

[13] B. Javidi, N. Towghi, N. Maghzi, S.C. Verrall, Appl. Opt. 39 (23) (2000) 4117.

[14] N. Towghi, B. Javidi, Z. Lou, J. Opt. Soc. Am. 16 (8) (1999) 1915.

[15] G. Unnikrishnan, K. Singh, Opt. Eng. 39 (11) (2000) 2853.

[16] G. Unnikrishnan, J. Joseph, K. Singh, Opt. Lett. 25 (12) (2000) 887.

[17] N.K. Nishchal, J. Joseph, K. Singh, Opt. Eng. 42 (2003) 1583.

[18] S. Liu, L. Yu, B. Zhu, Opt. Commun. 187 (2001) 57.

[19] Y. Zhang, C.H. Zheng, N. Tanno, Opt. Commun. 202 (2002) 277.

[20] B. Zhu, S. Liu, Opt. Commun. 195 (5-6) (2001) 371.

[21] B. Zhu, S. Liu, Opt. Lett. 26 (16) (2001) 1242.

[22] B. Zhu, S. Liu, Q. Ran, Opt. Lett. 25 (16) (2000) 1159.

[23] B. Hennelly, J.T. Sheridan, Opt. Eng. 43 (2004) 2239.

[24] B. Hennelly, J.T. Sheridan, Opt. Lett. 28 (4) (2003) 269.

[25] N.K. Nischal, G. Unnikrishnan, J. Joseph, K. Singh, Opt. Eng. 42 (12) (2004) 3566.

[26] B.M. Hennelly, J.T. Sheridan, Optik 114 (6) (2003) 251.

[27] O. Matoba, B. Javid, Opt. Lett. 24 (11) (1999) 762.

[28] G. Situ, J. Zhang, Opt. Commun. 232 (2004) 123.

[29] B.M. Hennelly, J.T. Sheridan, Proc. SPIE 2557 (2004) 233.

[30] G. Unnikrishnan, K. Singh, Opt. Commun. 193 (2001) 51.

[31] E. Wigner, Phys. Rev. 40 (1932) 749.

[32] M.J. Bastians, in: W. Mecklenbrauker, F. Hlawatsch (Eds.), The Wigner Distribution - Theory and Applications in Signal Processing, Elsevier, Amsterdam, 1997.

[33] J. Goodman, Introduction to Fourier Optics, second ed., McGraw-Hill, New York, 1996.

[34] H.M Ozaktas, Z. Zalevsky, M.A. Kutay, The Fractional Fourier Transform with Applications in Optics and Signal Processing, Wiley, New York, 2001.

[35] A.W. Lohmann, R.G. Dorsch, D. Mendlovic, Z. Zalevsky, C. Ferreira, J. Opt. Soc. Am. A 13 (1996) 470.

[36] A. Stern, B. Javidi, J. Opt. Soc. Am. A 21 (3) (2004) 360, Errata 21(10) (2004).

[37] S. Abe, J.T. Sheridan, J. Phys. A 27 (1994) 4179, Corrigenda in 7937.

[38] S. Abe, J.T. Sheridan, Opt. Lett. 19 (1994) 1801.

[39] M.J. Bastians, J. Opt. Soc. Am. 69 (1979) 1710. 